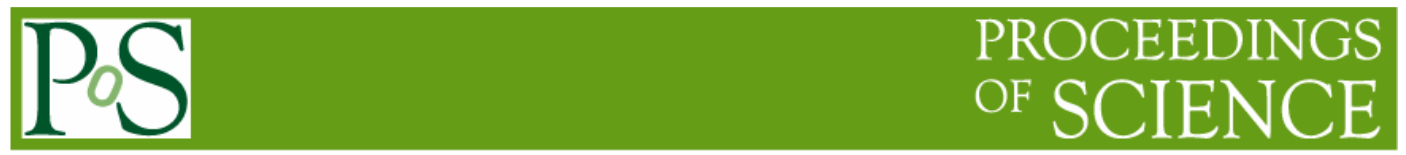

\title{
An Alidade for the SKA
}

\section{Bernard F. Burke ${ }^{1}$}

MIT

Room 37-641 Cambridge, MA, USA

E-mail: bfburkeespace.mit.edu

For the SKA, cost is a central consideration, and even small increments in cost per telescope in the array can be significant since there will be five or ten thousand elements. If the SKA is to operate at frequencies above $1 \mathrm{GHz}$, these will be steerable paraboloids. A small amount saved per element can amount to hundreds of millions (dollars or Euros) saved for the array. Here we examine only the alidade, which has historically cost about the same as the paraboloid that it supports. Scaling laws show that the cheapest design should have an alidade that has a broad base. An example of an alidade design is proposed that offers major reductions in the structural cost. More generally, it seems that very little research is being expended on optimizing steerable paraboloid design from a standpoint of cost. The general considerations are a reworking of material that has already been published by von Hoerner (1966).

From planets to dark energy: the modern radio universe

University of Manchester, Manchester, UK

1-5 October, 200

$1 \quad$ Speaker 


\section{The Alidade: Orders of Magnitude}

The alidade supports the elevation axis, and consists of the supporting structure that carries the forces and torques to the ground, the foundations, and the drive system. The dish, backup structure, and elevation drive should be the subject of a separate tradeoff study. The alidade structure should neither tip over nor undergo structural failure under survival wind conditions, but beyond this, for winds up to the operating wind velocity requirement, it must also maintain the specified pointing accuracy. It turns out that a sufficiently stiff structure to meet operational pointing requirements will also be secure against failure, with a substantial safety margin.

We first analyze the survival and pointing stiffness requirements for any alidade by applying basic physics. The height to the elevation axis will be a distance, which we will take to be $D / 2$, the radius of the dish. This is approximately the case for a paraboloid that can point to the horizon. The base will have dimension L, and the structure must carry the forces and torques to the ground. The wind force on the structure is the product of wind pressure $\mathrm{P}$ times the effective area of the resisting area $\mathrm{A}$, as shown in figure 1.

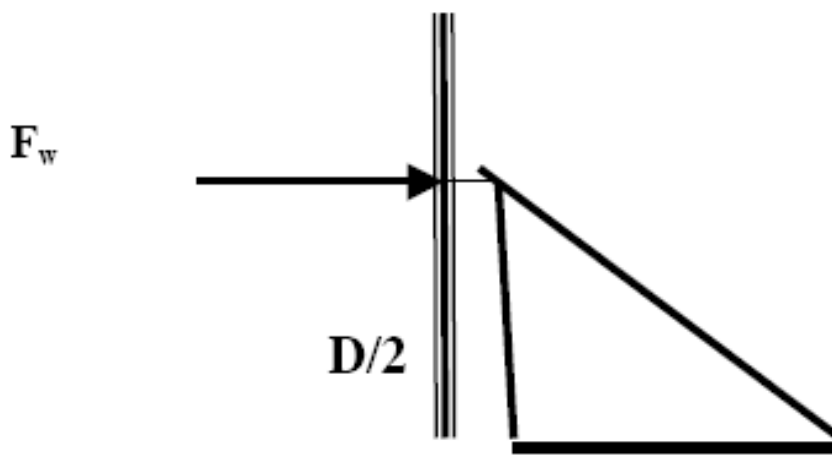

Figure 1 - force schematic

$\mathbf{L}$

This simplified diagram is all we need to estimate the orders of magnitude.

It is convenient to define a dimensionless parameter $y$, the ratio of the base to the height:

$$
\mathrm{y} \cong 2 \mathrm{~L} / \mathrm{D}
$$

Mixed units will be used: The price estimates will be for the US, so English units for construction and metric units for scientific specifications. A convenient scaling convention will be used: $\mathrm{D}_{10}$ is dish diameter in units of 10 meters, wind pressure $\mathrm{P}_{\mathrm{O}}$ in units of $40 \mathrm{lb} / \mathrm{sqft}$ ) corresponding to a wind velocity of about $100 \mathrm{mph}$ including the hydrodynamic factor $3 / 2$ (normal building design practice is $20 \mathrm{lb} / \mathrm{sqft}$ ), failure modulus $\mathrm{K}_{\mathrm{O}}$ in units of $4 \times 10^{4} \mathrm{lb} / \mathrm{sqft}$, and Young's modulus $Y_{O}$ in units of $3 \times 10^{7}$, both typical of low-carbon steel. First, consider the structural requirements for the alidade under survival condition. This leads to an estimate of $\sigma_{\mathrm{S}}$, the survival cross-section of the structural elements; $\sigma_{\mathrm{S}}$ must be of the form

$$
\sigma_{\mathrm{S}} \times \mathrm{PA} / \mathrm{K}
$$


There will be a dimensionless factor, $\mathrm{f}(\mathrm{y})$ that will depend upon the model, and a proportionality factor $g$ that will depend upon the units. In the present simplified example, $f(y)=\left(1+y^{2}\right)^{1 / 2} / y$. Using the previous default values,

$$
\sigma_{\mathrm{S}}=\operatorname{gf}(\mathrm{y})\left(\mathrm{P}_{\mathrm{O}} / \mathrm{K}_{\mathrm{O}}\right)(\pi / 4) \mathrm{D}_{10}^{2}
$$

or, in English units

$$
\sigma_{\mathrm{S}}=(0.785) \mathrm{f}(\mathrm{y})\left(\mathrm{P}_{\mathrm{O}} / \mathrm{K}_{\mathrm{O}}\right) \mathrm{D}_{10}{ }^{2} \mathrm{sq} \text { in }
$$

Survival is important, but the deflection of the structure under operating conditions is also a key consideration, in which case the wind pressure will be $\alpha \mathrm{P}_{0}$. In operation, the structure should not deflect by more than an angle that is some fraction $\varepsilon$ of the beam width. It follows that, if $\lambda_{1}$ is the minimum operating wavelength in centimeters, the cross-section of the element should be at least

$$
\sigma_{\mathrm{O}}=(1.05) \mathrm{f}^{\prime}(\mathrm{y})(\alpha / \varepsilon)\left(\mathrm{P}_{\mathrm{O}} / \mathrm{Y}_{\mathrm{O}}\right)\left(\mathrm{D}_{10}{ }^{3} / \lambda_{1}\right) \mathrm{sq} \text { in }
$$

If $(\mathrm{A} / \varepsilon \lambda)$ is of the order of 2 , a reasonable choice of parameters, it is the stiffness requirement, rather than the survival requirement, that determines the cross-section of the members unless $\mathrm{f}^{\prime}(\mathrm{y})$ is unusually small, a conclusion reached by von Horner long ago.

\section{The "Pyramid" Model}

A sketch of an alidade model that is easy to analyze is shown in figure 2 .

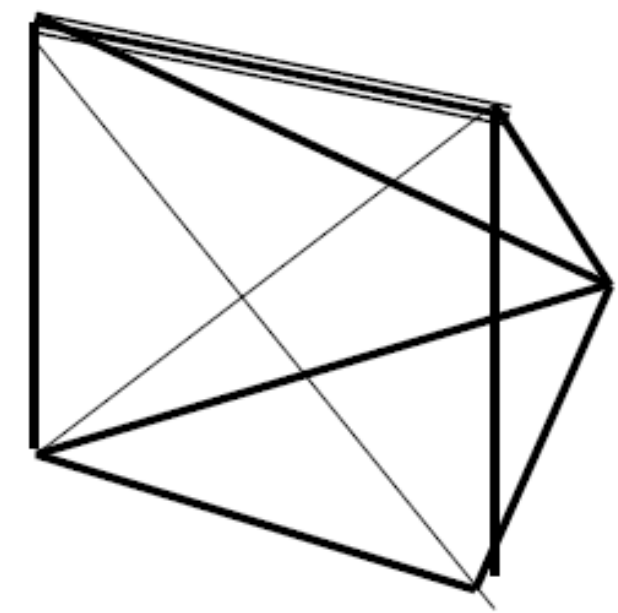

Figure 2 - Geometry of Structural Model 
The structure is not quite fully determined, since the face is square, with the elevation axis at the top, and because it is assumed that all vertices of the structure are pin joints, two tension rods are needed, running from the elevation bearings diagonally down to the lower opposite vertex. This will be called the "Pyramid" model, since it is a square pyramid lying on one of its triangular sides. This simple structure, using standard materials, should be cheap, and the fabrication costs should be easy to estimate.

If $\alpha=0.1,(30 \mathrm{mph}$ operating wind $)$ and $\varepsilon=0.05$, the operating cross-section for each brace must be more than three times the survival cross-section provided y is near unity and the diameter of the dish is approximately $10 \mathrm{~m}$. The operating requirement, therefore, guarantees that the survival requirement will be met, with a substantial safety factor. For the SKA, an alidade structure that meets reasonable pointing constraints will be safe from catastrophic wind failure,

Note that the deflection cost scales like $\mathrm{y}^{-2}$. A narrow base inflicts a fearsome penalty in pointing stability, and this explains the great mass required for the alidade structure in the current, ATA-derived design in the US proposal.

\section{Practical Matters}

The proposed structure would be built from standard steel pipe, which could be 8-inch diameter, 1/4-inch wall welded steel pipe, a standard size. The ratio of member length to radius of gyration would be of the order of 50, which would be safely below the buckling limit and within standard engineering practice. The cross-section of the metal in the pipe would keep the deflections down to .03 beamwidths for a $10-\mathrm{m}$ diameter dish, and if the dish diameter were to be $12 \mathrm{~m}$, the deflection would be .06 beamwidths, probably still an acceptable requirement.

At the vertices, the pipes must be tied together. The three base vertices could be steel weldments that receive the structural pipes and the azimuth drive wheels, which will be three standard 20inch railroad wheels. A detailed engineering study is needed to determine the exact configuration; the wheel bearings, drive motor mounts, and the attachments to the structural pipes present standard engineering problems. The attachments could be standard flanges, or locating sleeves to support the pipes for field welding. A preliminary estimate is that these three mounting plates would weigh about 300 pounds each, while the junctions for the elevation axis bearings, with attachment points for the uprights, braces, and tension rods might be about 200 pounds each.

With a standard railroad wheel at each vertex of the base triangle, the location of the structure on the azimuth track is indeterminate. This requires a standard pintle bearing, with spokes to the three base vertices that are sturdy enough to carry the lateral wind forces, and the location of the structure is thus determined. The support wheels must not scrub and gall, and the proposed design, being kinematic, should avoid that problem. The proposed dish structure is not heavy, less than 10,000 lb, and railroad technology is highly developed. Railroad tracks have a curved profile, as do railroad wheel flanges, and when the track is properly laid, there is no screech from the wheels scrubbing on the tracks. Here we propose a circular track, probably of $200-\mathrm{lb}$ rail, laid accurately enough so that radial and vertical variations are small, with a precision of $0.02-0.04$ inches or so, a relatively crude tolerance by millwork standards.

The configuration of three wheels on railroad track, tied to a central pintle bearing, gives a system that is self-locating, and the wheel loadings would be modest. The drive moment arm of approximately 8 to 10 feet is sufficiently large so that relatively small drive motors and gear reducers could be used. There would have to be hold-down brackets, since the dish is light, and 
the weight of the dish must be sufficient to keep it from rocking in conditions within the operating wind specification. There has to be a locking mechanism to keep the dish from pinwheeling under survival conditions; whether these are railroad shoe-brakes of a conventional sort, or built into the hold-down brackets, would be determined by a detailed design.

\section{Some Cost Estimates}

The cost of welded steel pipe with flanges is remarkably low if standard pipe is used. A rough estimate of the present cost has been obtained from Larry Jones of the LIGO project, who says that the steel will run 25 to 40 cents per pound, depending on the country of origin, and fabrication would be in the range 25 to 60 cents per pound. Thus the pipes would cost about $\$$ 0.50 to $\$ 1.00$, per pound, plus shipping.

We now scale the weight for a 12 -meter dish, keeping $\mathrm{y}=1$. If we keep to $1 / 4$-inch wall, 8 -inch diameter pipe, the operating pointing accuracy will be 0.035 beamwidths, still an acceptable number. There will be six elements 6 meters long, including the elevation axis (about 20 feet each, which we take as a good enough approximation for the present), and two braces $\sqrt{ } 2$ longer (say 30 feet), giving a total weight of the seven pipes in the structure of approximately $3600 \mathrm{lb}$. The three spokes from the pintle bearing will add $700 \mathrm{lb}$. and the two steel tension rods, which can be 1 1/2 -inch steel bars, add another $800 \mathrm{lb}$.; thus, taking the top cost estimate of $\$ 1$. per pound, the structural elements will cost no more than about $\$ 5100$ plus shipping. We estimate the shipping cost at $\$ 0.50$ per pound (depends on origin), which gives $\$ 7700$ for pipes, rods, and shipping. Fastenings should cost no more than $\$ 1000$. The weldments at the 5 vertices and at the pintle bearing will weigh a total of $1400 \mathrm{lb}$., and these will cost more to fabricate; we take the estimate of the USSKA proposal of $\$ 1.70$ per pound, giving $\$ 2400$ for the junction weldments. The structure must be assembled, and since it is a kinematic structure, assembled by bolting flanges together, it is a standard steel-construction job. A crew of 4 should be able to assemble it in a day, at a cost (with overhead) of perhaps $\$ 2000$, using the labor estimate of $\$ 60$ per manhour hour given in the USSKA proposal. The cost of motors, reducers, and bearings might come to $\$ 10,000$. Thus, the total alidade structure should cost about $\$ 22,100$, including azimuth drive and bearings.

Cost estimate for the foundation pad and track follow. If 200-lb rail (200 lb per10-ft length) is used, the 123-foot circumference of track will weigh $2500 \mathrm{lb}$, and cost $\$ 2500$ when fabricated into a circle. There is an extra cost item in fastening the rail to the foundation; one should probably double this cost to allow for the fastening system.

The foundation would be reinforced concrete, at \$200. per cubic yard including rebar work, the standard cost for foundation-work in Massachusetts. For concrete weighing $137 \mathrm{lb}$ per cubic foot, or 1.8 short tons per cubic yard, a 60-ton foundation would require 33 cubic yards of concrete. Excavation cost and setting the anchors for the track are estimated at $\$ 4000$. Thus the reinforced concrete foundation and the azimuth track would cost about $\$ 14600$ including labor.

The total cost of foundation and alidade, with azimuth drive, comes to $\$ 36,700$ for a 12 -meter dish. It does not appear that an alidade can be much cheaper than this, unless major labor savings can be found.

The work on low-cost, hydroformed dishes for the ATA, or the investigations of other fabrication methods at JPL, will probably result in workable reflector surfaces that are unlikely to be improved upon. If the final cost of a $12-\mathrm{m}$ dish is $\$ 50,000$, and the elevation drive, angle 
indicators, computers, and motor controllers can by obtained for $\$ 20,000$, the total cost, installed, of each element should be $\$ 107,000$, according to the above estimates.

One remark is in order concerning the size of each dish. There is a modest gain in alidade cost per element if the size is reduced. Going down in size from 12 to $10 \mathrm{~m}$, for example, gives a cost reduction of 1.7, while the collecting area is reduced by 1.44 , so the net cost per unit collecting area improves (the mass of the alidade scales like $\mathrm{D}_{10}{ }^{3}$, while the collecting area scales like $\mathrm{D}_{10}{ }^{2}$ ). Since more receivers etc. are needed, there does not seem to be much net gain; the cost minimum usually turns out to be shallow.

The main uncertainty is in the dish cost. One might expect it to scale the same way as the alidade, but since novel fabrication procedures are used, this might not be the case. One will only find out, probably, by manufacturing a $12-\mathrm{m}$ model. As far as the alidade is concerned, the exact size is not a fabrication concern, so the size decision depends upon the dish fabrication.

\section{Conclusion}

The estimate given above for a straightforward rail-and-track alidade, if the estimates hold up under examination, indicate that the cost per element of the US small $\mathrm{D}$, large $\mathrm{N}$ concept is not likely to be much less than $\$ 110,000$ per element. Perhaps the number will come down some when manufacturing experts examine cost-cutting possibilities, but probably not by a major factor. If there are $500012-\mathrm{m}$ elements in the SKA, their cost will be approximately $\$ 550$ million.

There should be a close examination of the comparative advantages of the ATA king-post design with other concepts. The ATA design does not have as stringent a stiffness requirement as that given here, and since the stiffness cross-section scales like $\mathrm{y}^{-2}$ scaling the design up in size may lead to problems that should be examined. It is in order, also, to examine the assumption that no foundations are required, the post being sufficiently well-stuck in the dirt to resist tipping, although it can be tied to bedrock if it is not too deep. Having observed many leaning telephone poles, it can reasonably be asked what sort of soil studies are needed, and how variable the soil and rock conditions are over the hundreds or thousands of kilometers covered by the SKA site.

The pyramid design presented here uses a rail-and-track azimuth drive, and the experience with the GBT has given the concept a bad name. The difficulties that were encountered, to put it bluntly, were caused by poor engineering. The pyramid design should be free of such difficulties, since the loadings are light and proper attention to engineering practice should result in a highly reliable telescope. There is no substituting for actual experience, and an engineering model should be built, or at least a model independent of the current ATA design should be properly tested. Given the large sums that are involved in antenna manufacture and installation, it would seem only prudent to pursue alternative designs. Since the alidade constitutes such a large fraction of the total, it is particularly in need of an intensive and creative engineering effort.

\section{References:}

Sebastian von Hoerner, "Design of Large Steerable Antennas" Astron.J.J. 72, 35 (1967) 